\title{
Hebammer oder Hebammerich - bisher eine Rarität
}

\author{
Pioniere im Kreißsaal Tobias und Raffaele sind die einzigen zwei Entbindungs- \\ pfleger - so ist die offizielle Berufsbezeichnung für männliche Vertreter des \\ Berufsstandes der Hebammen - am Helios Klinikum Berlin-Buch. Und auch im \\ Rest der Republik sind sie eine Rarität. Gerade einmal zehn ausgelernte männ- \\ liche Hebammen gibt es laut Deutschem Hebammenverband momentan hierzu- \\ lande, darunter einige Italiener.
}

$\mathbf{E}$ s gibt Frauenärztinnen und männliche Gynäkologen. Es gibt Krankenschwestern und Krankenpfleger. Und es gibt Hebammen und - ja, wie heißen sie eigentlich, die männlichen Exoten, die in dieser Frauendomäne arbeiten? Tobias Richter erklärt, dass er und seine männlichen Kollegen sich auch Hebammen nennen und das auch offiziell dürfen, weil viele Frauen mit dem Begriff Entbindungspfleger nichts anfangen können, oft sogar bei der Vorstellung ausschließlich pflegerische Tätigkeiten mit ihnen assoziiert haben. „Viele Frauen wussten einfach nicht, dass wir das komplett gleiche machen wie Hebammen. Seitdem wir uns Hebamme nennen, gab es dieses Problem nie wieder", sagt er sichtlich abgekämpft aber glücklich, als er in der Elternschule des Helios Klinikums Berlin-Buch sitzt, die er leitet. Er kommt geradewegs aus dem Kreißsaal, wo er vor wenigen Minuten - genau zum Ende seiner Schicht - noch einem Baby auf die Welt geholfen hat. Raffaele Mirabile, die zweite männliche Hebamme in Buch, sitzt neben ihm und gibt zum Besten, dass er sogar schon einmal „Hebammer" genannt wurde; aus Spaß wurden sie auch schon „Hebammerich“ betitelt, weiß Tobias. Die gelöste Stimmung täuscht fast darüber hinweg, dass es die männlichen Hebammen in Deutschland immer noch schwer haben und um ihre Akzeptanz kämpfen müssen.

\section{Eine schwere Geburt}

In Italien gehört das Bild der männlichen Hebamme zum Alltag an Kliniken. An jedem Krankenhaus im Nachbarland findet man durchschnittlich ein bis zwei männliche Hebammen auf der Entbindungsstation. „In Italien ist es ganz normal, dass Männer als Hebammen arbeiten“, erzählt Raffaele erstaunt darüber, dass es in Deutschland nur so wenige gibt. Er selbst hat auf Sizilien den Beruf studiert und ist dann nach einem Jahr im Beruf nach Deutschland gegangen. „Auch wenn ich in Italien als Hebamme Ultraschall, Krebsvorsorge und andere Dinge machen darf, bekomme ich hier die Möglichkeit, viele schöne neue Erfahrungen zu machen und mich zu verwirklichen. "In Italien gäbe es generell viel weniger Heb- ammen an den Krankenhäusern als in Deutschland und viele erlernen den Beruf. Da es im Land von Vino und Pasta eine hohe Arbeitslosigkeit unter Hebammen gebe, biete es sich an, nach Deutschland zu kommen, wo man händeringend nach Verstärkung sucht.

Während in Australien, Dänemark oder eben Italien Männer selbstverständlich in diesem Beruf arbeiten, scheint es in Deutschland eine schwere Geburt zu werden, bis Männer gleichrangig in dem Beruf tätig werden. Hierzulande üben aktuell gerade einmal zehn Männer den Beruf aus - und das, obwohl es viel zu wenige Hebammen gibt. Die Zahl der Bewerbungen nehme laut Tobias zu. Sachte scheint sich eine Veränderung anzubahnen. Mit der Akademisierung des Berufs könnte eine größere Akzeptanz und Attraktivität des Hebammenberufs - auch für Männer - einhergehen, hofft er. Schließlich sind männliche Hebammen in anderen Ländern, wo das Hebammenwesen ähnlich wie die Pflege akademisiert ist, etwas ganz Normales.

\section{Noch fehlende Offenheit}

Gründe für die geringe Anzahl männlicher Hebammen sieht Tobias darin, dass die wenigsten wissen, dass die Ausbildung auch von Männern absolviert werden könne. Pflegerische und soziale Berufe seien zudem nicht so gut bezahlt - das finden Männer häufig unattraktiv. Zudem fehle in der Gesellschaft die Offenheit. „Viele Frauen nehmen ihre Männer ja noch nicht einmal zum Geburtsvorbereitungskurs mit. Viele kommen mit dem Thema Geburt einfach gar nicht in Berührung“, weiß er zu berichten. Ganz anders war es bei ihm. Schon seine Mutter war Hebamme und er ist mit dem Beruf groß geworden. „Ich wusste schon sehr früh, dass ich Hebamme werden wollte“, erklärt er.

Bereits sein Schulpraktikum hat er im Krankenhaus absolviert und ein Pflegepraktikum auf der Inneren Station. „Einen Praktikumsplatz im Kreißsaal zu bekommen war gar nicht so einfach. Daher bin ich über die Umwege gegangen. Da habe ich dann aber auch schnell gemerkt, dass Gesundheits- und Krankenpflege nicht 


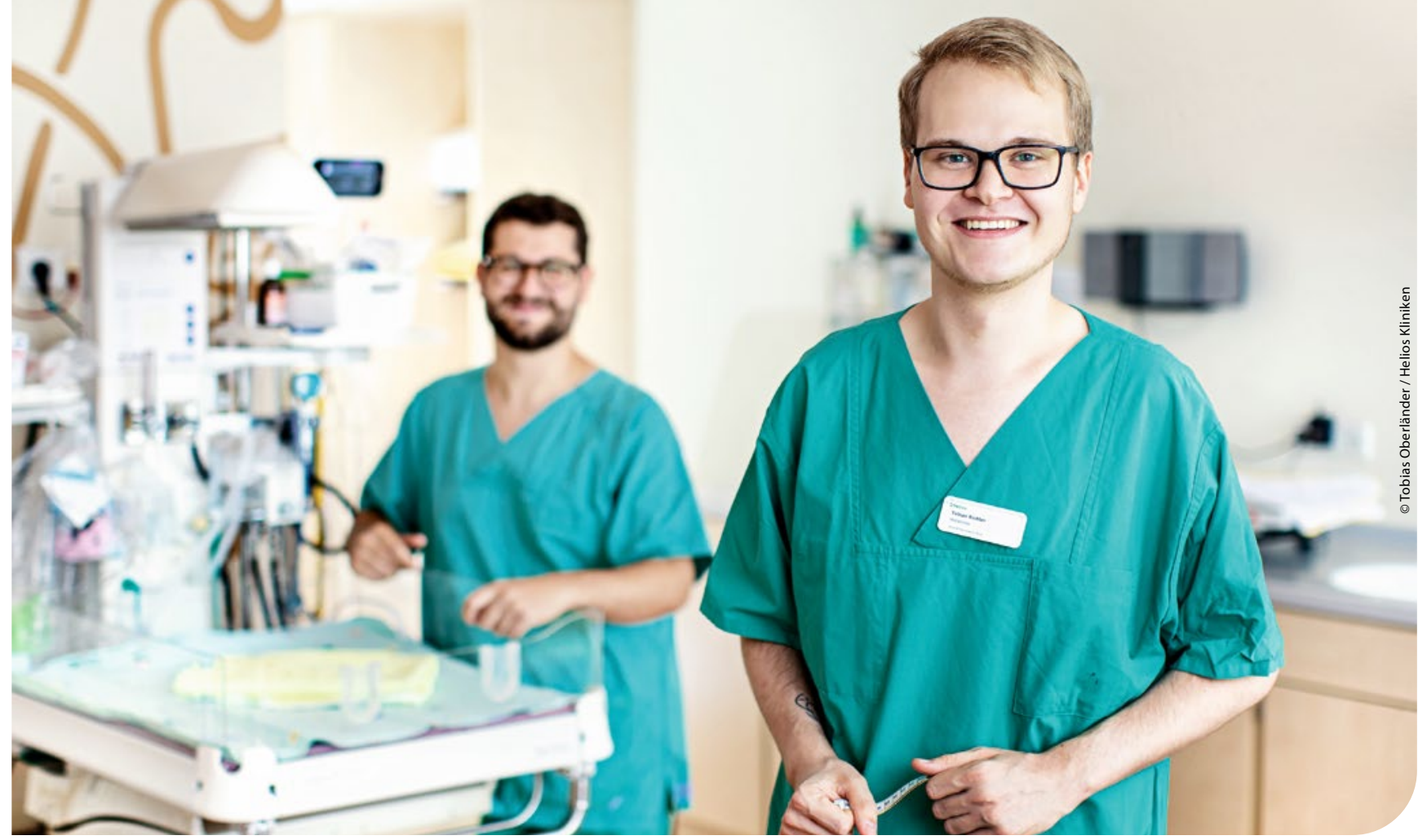

Tobias (vorn) und Raffaele - allein unter Frauen. Die beiden männlichen Hebammen sind bisher eher eine Ausnahme. Mit werdenden Müttern und Vätern hatten sie noch nie wirklich Probleme, höchstens mal ein Stirnrunzeln.

mein Ding ist“, erklärt die gestandene Hebamme. „Als ich hier im Haus ein Praktikum im Kreißsaal machen durfte, war klar, dass ich genau das machen will. Danach habe ich mich für die Hebammenausbildung beworben und wurde nach unzähligen Anläufen in Erfurt genommen“, berichtet Tobias von den Anfangsschwierigkeiten. Vierzig Bewerbungen musste er schreiben, bis er einen Ausbildungsplatz hatte. In Thüringen war er dann die erste männliche Hebamme an der Schule überhaupt und sorgte am ersten Ausbildungstag für Irritationen - sowohl auf Lehrerseite als auch bei seinen Mitschülerinnen. „Wir haben uns aber schnell aneinander gewöhnt“, sagt Tobias - auch wenn er gerade am Anfang viel reden und von sich erzählen musste. „Im Kreißsaal hätte ich mir dagegen etwas mehr Verständnis von den Kolleginnen gewünscht.“ Es habe Kolleginnen gegeben, die nicht mit ihm arbeiten wollten.

\section{Männer werden unterschätzt}

Frauen in Männerberufen sind auf dem Vormarsch. Aber wie sieht es andersrum aus? Tobias ist 22 Jahre und auch Raffaele ist mit Mitte 20 noch sehr jung. Beide erzählen, dass die meisten Kolleginnen und Kollegen - und auch Patientinnen - positiv auf sie reagieren. Aber sie haben auch das Gefühl, dass sie sich mehr beweisen müssen als ihre Hebammen-Kolleginnen. „Es gibt auch einige, die darauf warten, dass man Fehler macht. Daher müssen wir uns besonders beweisen“, erklärt er. Sie können nur mit viel Empathie in ihrer Arbeit überzeugen und mit ihrer verständnisvollen Seite punkten. „Wenn Frau Müller aus Posemuckel einen Fehler macht, dann ist das so. Wenn wir Mist bauen, dann wissen das alle gleich, weil wir männliche Hebammen im Fokus stehen - im Team, aber auch extern. Und viele sehen sich dann darin bestätigt, dass wir als Mann den Beruf sowieso nicht richtig ausüben können“, so Tobias.

Wenn Raffaele und er damit konfrontiert werden, dass sie als Männer nicht wüssten, wie es sich anfühle, ein Kind zur Welt zu bringen, dann haben sie eine klare Antwort: „Wenn Sie sich das Bein gebrochen haben, hilft es Ihnen nicht, wenn der Chirurg schon mal eine Fraktur hatte. Es ist gerade bei der Geburt wichtig, Einfühlungsvermögen und Empathie zu haben. Und das ist unabhängig von der Chromosomenkonstellation der Hebamme." Und obwohl weder Raffaele noch Tobias selbst gestillt haben, wissen sie natürlich, was bei einem Milchstau zu tun ist. Denn auch das haben sie in ihrer Ausbildung gelernt. Genauso wie ihre kinderlosen Kolleginnen. Sie arbeiten beide in ihren Traumberufen. „Ich bin professionell dabei, da spielt das Geschlecht keine Rolle. Es ist toll, dass das in Italien schon so ist. Ich bin aber sicher, dass das hierzulande auch noch Normalität wird“, erklärt Raffaele, dem hier in Berlin ab und an das Meer fehlt. Aber die Flugverbindungen nach Sizilien sind hervorragend, so dass er alle drei Monate die Familie, Freunde und das Meer besuchen kann.

\section{Geburten - keine wie die andere}

Das ist das stolze Ergebnis von Tobias bis zum heutigen Tag. Dabei war keine wie die andere. „Wenn man dann ein Kind nach der Geburt in den Händen hält, dann ist das jedes Mal ein wahnsinnig schöner Moment", führt Tobias aus. Er liebe Kinder und habe ein Händchen für die Babys, sagen auch seine Kolleginnen in Buch.

Schon während seiner Ausbildung hat er sich berufspolitisch in der Arbeitsgemeinschaft "Junge Hebammen“ engagiert. Er ist stolz, dass die Hebamme (m/w/d) auch ein bisschen mit auf seine Initiative entstanden ist. In Deutschland sei der Hebammenmangel aus seiner Sicht ein hausgemachtes Problem. Mit dem Studium sei etwas in Richtung Attraktivität des Berufsbildes getan worden, nun aber müsse auch finanziell nachjustiert werden. Und dass Hebammen nicht als systemrelevant eingestuft wurden, sei für das Berufsbild auch nicht gerade förderlich. Denn Corona macht nicht vor dem Kreißsaal halt.

>II

Alexandra Heeser 